\title{
Persistência da ação inseticida e repelência da Terra de Diatomácea \\ para o cascudinho-dos-aviários Alphitobius diaperinus \\ (Panzer, 1797) (Coleoptera: Tenebrionidae)
}

\author{
Persistence of insecticidal activity and repellency of the \\ diatomaceous earth against the lesser mealworm Alphitobius \\ diaperinus (Panzer, 1797) (Coleoptera: Tenebrionidae)
}

\author{
Daian Guilherme Pinto de Oliveira ${ }^{1 *}$; Luis Francisco Angeli Alves ${ }^{2,3}$; \\ Luiz Paulo Calixto Marchese ${ }^{2,4}$; Dhyego Thomazoni ${ }^{2}$
}

\section{Resumo}

\begin{abstract}
A persistência da ação inseticida da terra de diatomácea (TD), e o seu efeito repelente foram avaliados contra o cascudinho-dos-aviários, Alphitobius diaperinus. Para o experimento de persistência foram utilizados potes plásticos $\left(20 \mathrm{~cm}\right.$ de diâmetro) com cama tratada com TD $\left(172 \mathrm{~g} / \mathrm{m}^{2}\right)$. Foram liberados $13 \mathrm{~g}$ de adultos de cascudinho (cerca de 1100 insetos), e a mortalidade foi avaliada retirando-se e fazendo a contagem dos insetos mortos nos recipientes 10, 20 e 30 dias após a infestação. Este procedimento repetiu-se com 80,160,240, 320 e 400 dias após a aplicação de TD. Para avaliação da repelência foram utilizadas bandejas plásticas com $(36 \times 24 \times 6 \mathrm{~cm} \mathrm{C} \times \mathrm{L} \times \mathrm{A})$, contendo $5 \mathrm{~cm}$ de cama de aviário tratada e não-tratada com TD $\left(200 \mathrm{~g} / \mathrm{m}^{2}\right) \mathrm{em}$ metade de cada bandeja. Foram liberados $13 \mathrm{~g}$ de adultos de cascudinho na linha de divisão dos tratamentos, avaliando-se após 1, 4, e 7 dias da infestação, pela contagem dos insetos presentes em cada área. Obteve-se atividade inseticida até oito meses após a aplicação (60\% de mortalidade), demonstrando a persistência da ação inseticida da TD na cama de aviário. Além disso, o produto apresentou ação repelente para os insetos, a qual deve ser considerada no estabelecimento de estratégias de controle do cascudinho.
\end{abstract}

Palavras-chave: Controle alternativo, pós-inertes, produção animal, TD

\begin{abstract}
The persistence and repellency of the diatomaceous earth (DE) formulation was evaluated against the lesser mealworm, Alphitobius diaperinus. Persistence bioassays werw conduced in $20 \mathrm{~cm}$ diameter plastic cups with DE-treated poultry litter $\left(172 \mathrm{~g} / \mathrm{m}^{2}\right)$. The adults were transferred $(13 \mathrm{~g}$ - about 1100 insects) and the mortality was assessed by counting and removing dead adult beetles in the cups 10 , 20 and 30 days after placement. All procedures were repeated 80,160, 240, 320 and 400 days after first evaluation. The repellency test was conducted in plastic box $(36 \times 24 \times 6 \mathrm{~cm} \mathrm{~L} \times \mathrm{W} \times \mathrm{H})$ containing poultry litter trated and non-treated with $\mathrm{DE}\left(200 \mathrm{~g} / \mathrm{m}^{2}\right)$, distribuited in two parts. The adults were added to the litter $(13 \mathrm{~g})$, and the evaluation ocurred after 1, 4 and 7 days after placement, by counting

$1{ }^{1}$ Escola Superior de Agricultura 'Luiz de Queiroz'-ESALQ/USP, Departamento de Entomologia, Fitopatologia e Zoologia Agrícola, Av. Pádua Dias, 11, Piracicaba, SP. E:mail: daianguilherme@yahoo.com.br

$2{ }^{2}$ Universidade Estadual do Oeste do Paraná, Campus de Cascavel, CCBS, Laboratório de Zoologia de Invertebrados, Rua Universitária, 2069, Cascavel, PR.

$3{ }^{3}$ *Bolsista de Produtividade em Pesquisa pelo Conselho Nacional de Desenvolvimento Científico e Tecnológico (CNPq), E-mail:

$4{ }^{4}$ Bolsista de Iniciação Científica, PIBIC/CNPq/Unioeste.

Autor para correspondência
\end{abstract} lfaalves@unioeste.br 
the insects in the treated and non-treated area. It was verified the inseticidal activity until eight month after litter treatment ( $60 \%$ of mortality), showing the persistence of the product. The repellency activity of the DE was confirmed and it must be considered in a development of control strategies of the lesser mealworm with.

Key words: Alternative control, inert dust, animal production, DE

\section{Introdução}

O besouro Alphitobius diaperinus (Panzer, 1797) (Coleoptera: Tenebrionidae) é considerado uma importante praga avícola de ocorrência mundial. Vive nos aviários onde se abriga sob a cama e alimenta-se de ração e carcaças de aves (DESPINS; AXTELL, 1995). Ocasiona prejuízos, pois as aves se alimentam destes insetos, deixando de ingerir a ração, causando conseqüente redução na conversão de peso (CHERNAKI-LEFFER et al., 2001). Além disso, causa danos estruturais de até $30 \%$ no material isolante utilizado nos aviários de regiões de clima frio (STEELMAN, 1996). Também pode ser potencial vetor de inúmeras doenças causadas por vírus, bactérias, fungos, protozoários e helmintos, uma vez que alguns microorganismos já foram isolados deste inseto (BATES; HIETT; STERN, 2004; CHERNAKI-LEFFER et al., 2002; MCALLISTER et al., 1995).

Atualmente, buscam-se alternativas para o controle do cascudinho, visando reduzir a utilização dos inseticidas químicos, que apesar de eficientes apresentam problemas, como a seleção de indivíduos resistentes, risco de intoxicação do ambiente, aves e homem, além de eliminarem os inimigos naturais.

Dentre as alternativas, destacam-se no Brasil, estudos que mostram o potencial do extrato de neem (Azadiracta indica), nematóides e, principalmente, fungos entomopatogênicos (ALVES; ALVES; ROHDE， 2005; CHERNAKI-LEFFER， 2004; MARCOMINI, 2006; ROHDE et al, 2006).

Contudo, Chernaki-Leffer et al. (2001) também sugerem o uso de pós-inertes à base de terra de diatomácea (TD), sendo confirmado em laboratório e em campo, que tal produto apresenta elevada ação inseticida (ALVES et al., 2006), e também a possibilidade da associação deste produto com fungos entomopatogênicos para o controle da praga (OLIVEIRA; ALVES, 2007; SANTORO et al., 2008).

A TD é obtida em jazidas de algas diatomáceas fossilizadas, constituídas predominantemente por dióxido de sílica amorfa, sendo utilizada no controle de pragas de grãos armazenados há bastante tempo (KORUNIC, 1998; QUARLES, 1992).

Seu modo de ação se dá pela desidratação ou dessecação, uma vez que partículas do pó aderem ao corpo dos insetos e ocorre a remoção da cera epicuticular, devido à abrasão no tegumento ou a adsorção. Assim, o inseto perde água excessivamente e morre (KORUNIC, 1998). O produto é atóxico, seguro para pessoas, animais e ambiente, sendo utilizado para o controle de pragas de grãos armazenados e tem a vantagem de não promover a resistência em insetos (QUARLES, 1992; LORINI et al., 2001).

Segundo Quarles (1992), a TD tem ação inseticida duradoura, e não perde a eficácia ao longo do tempo. Contudo, estudos recentes em ambiente de armazenamento de grãos, mostraram que os produtos comerciais à base de TD possuem a persistência dependente de inúmeros fatores bióticos e abióticos, e apesar de duradoura, ocorre sim, perda da eficácia depois de determinado tempo (ATHANASSIOU; KAVALLIERATOS; ADRIS, 2004; STATHERS; MVUMI; GOLOB, 2002).

Além da ação inseticida, a TD pode apresentar efeito repelente, dependendo da concentração utilizada, conforme observado para três espécies pragas de grãos armazenados, Sitophilus oryzae (Linnaeus, 1763) (Coleoptera: Curculionidae), Tribolium castaneum (Herbst, 1797) (Coleoptera: 
Tenebrionidae), Cryptolestes ferrugineus (Stephens, 1831) (Coleoptera: Laemophloidae). (MOHAN; FIELDS, 2002). Pinto Junior (1999) avaliou o efeito repelente da TD para ninfas e adultos de Blatella germanica, e também constatou este efeito para os adultos do inseto. No entanto, não existem trabalhos sobre estes possíveis efeitos da TD para o cascudinho.

A persistência da TD na cama de aviário é um fator preponderante para o estabelecimento de estratégias de sua utilização para o controle do cascudinho em condições de campo, uma vez que se possibilita estimar o intervalo entre as aplicações do produto. Da mesma forma, é importante avaliar a resposta do inseto em relação aos locais de aplicação, sendo estes os objetivos do presente estudo.

\section{Material e Métodos}

Os experimentos foram realizados no Laboratório de Zoologia de Invertebrados da Universidade Estadual do Oeste do Paraná, com insetos provenientes de aviários comerciais da região de Cascavel, PR, que foram trazidos para o laboratório e mantidos em baldes plásticos fechados com tampa perfurada, contendo cama de aviário e alimentados com ração para aves, com temperatura de $26 \pm 2{ }^{\circ} \mathrm{C}$ e fotoperíodo natural.

$\mathrm{O}$ produto à base de TD utilizado para os testes foi fornecido pela empresa Keepdry (Keepdry ${ }^{\mathrm{TM}}$ ), contendo $86 \%$ de dióxido de sílica amorfa e com granulometria média de até $5 \mu \mathrm{m}$.

\section{Persistência da TD na cama de aviário}

Foram utilizados potes plásticos de $20 \mathrm{~cm}$ de diâmetro contendo $5 \mathrm{~cm}$ de cama usada, a qual foi previamente coletada no aviário, trazida para o laboratório e retirados todos os insetos. A TD foi utilizada na concentração de $172 \mathrm{~g} / \mathrm{m}^{2}$, conforme Alves et al. (2006), polvilhada sobre a cama de aviário, e incorporada por meio de uma espátula metálica, e em seguida foi acrescentada ração para frangos na superfície da cama. A testemunha constou de recipientes contendo apenas a cama de aviário usada e a ração para a alimentação dos insetos.

Em seguida, foram liberados $13 \mathrm{~g}$ de adultos por pote (aproximadamente 1100 insetos), sendo realizadas cinco repetições para cada tratamento. Os recipientes foram fechados com tampa perfurada e acondicionados em câmara tipo BOD a $26 \pm 2^{\circ} \mathrm{C}$ e fotofase de 14 horas.

A avaliação da mortalidade dos insetos foi realizada aos 10, 20 e 30 dias após a infestação, distribuindo-se todo o conteúdo de cada recipiente em uma bandeja, capturando e contando os insetos mortos e devolvendo-se os vivos aos respectivos potes.

Com o término do experimento (30 dias), após a ultima avaliação, todos os insetos restantes foram retirados dos potes e descartados. O procedimento de liberação dos insetos e avaliação por 10, 20 e 30 dias repetiu-se com 80, 160, 240, 320 e 400 dias após o tratamento da cama, pois normalmente em condições de campo, a cama permanece por oito lotes (1 ano) no aviário.

Os dados de mortalidade obtidos foram previamente transformados em $\operatorname{arcsen} \sqrt{\mathrm{x} / 100}$ e submetidos à análise de variância no programa Sisvar ${ }^{\circledR} \quad$ (FERREIRA, 1992), utilizandose o delineamento experimental inteiramente aleatorizado, com parcelas subdivididas no tempo (split-plot), as quais corresponderam aos dias após o tratamento (DAT), comparando-se as médias pelo teste de Tukey $(\mathrm{P}<0,05)$.

\section{Repelência}

Para a avaliação da repelência da TD foi utilizada cama de aviário, sendo retirados anteriormente todos os insetos presentes neste substrato. Foram utilizadas bandejas plásticas retangulares com $24 \mathrm{~cm}$ (largura) $\times 36 \mathrm{~cm}$ (comprimento) $\times 6 \mathrm{~cm}$ (altura), contendo cama usada de aviário ( $5 \mathrm{~cm}$ de profundidade). As 
bandejas foram divididas ao meio, sendo uma das metades tratada com TD $\left(172 \mathrm{~g} / \mathrm{m}^{2}\right)$ e outra parte não tratada. Após a aplicação, ambas as partes receberam $28 \mathrm{~g}$ de ração para aves espalhados na superfície, e em seguida, foram liberados $13 \mathrm{~g}$ de insetos adultos de cascudinho (aproximadamente 1100 indivíduos) na linha de divisão dos tratamentos.

Os recipientes foram cobertos com tecido para permitir a circulação de ar e mantidos em sala climatizada à temperatura ambiente $\left(26 \pm 2^{\circ} \mathrm{C}\right)$, sendo preparadas três repetições para cada tratamento. As bandejas foram avaliadas depois de 1, 4, e 7 dias da liberação dos insetos, e a umidade da cama foi determinada segundo a metodologia da EMPRESA BRASILEIRA DE PESQUISA AGROPECUÁRIA - EMBRAPA (1997).
Os dados de mortalidade obtidos foram previamente transformados e analisados adotandose o mesmo procedimento descrito anteriormente, visando confrontar o número de insetos nas áreas tratada enão tratada, ao longo do tempo, comparandose as médias pelo teste de Tukey $(\mathrm{P}<0,05)$.

\section{Resultados e Discussão}

\section{Persistência da TD na cama de aviário}

Observou-se que a eficácia da TD mantevese superior a $60 \%$ até 270 dias após o tratamento da cama, sendo reduzida a partir de 320 dias, e mantendo-se baixa também na avaliação com 400 dias (Tabela 1).

Tabela 1. Porcentagem de mortalidade de Alphitobius diaperinus em uma mesma cama de aviário tratada com Terra Diatomácea após diferentes períodos de exposição $\left(26 \pm 2^{\circ} \mathrm{C}, 14 \mathrm{~h}\right.$ de fotofase).

\begin{tabular}{ccccc}
\hline \multirow{2}{*}{$\begin{array}{c}\text { Dias após o } \\
\text { tratamento }\end{array}$} & \multicolumn{4}{c}{ Tempo de exposição (Dias) } \\
\cline { 2 - 5 } & 10 & 20 & 30 & Total \\
\hline 0 & $5,7 \pm 0,88 \mathrm{~B} \mathrm{c}$ & $43,0 \pm 6,50 \mathrm{~A} \mathrm{a}$ & $32,0 \pm 2,51 \mathrm{~A} \mathrm{~b}$ & $80,7 \pm 2,66 \mathrm{~A}$ \\
80 & $6,3 \pm 2,02 \mathrm{AB} \mathrm{b}$ & $28,7 \pm 2,64 \mathrm{~B} \mathrm{a}$ & $28,1 \pm 2,88 \mathrm{~A} \mathrm{a}$ & $63,1 \pm 3,28 \mathrm{~B}$ \\
160 & $13,4 \pm 2,33 \mathrm{~A} \mathrm{~b}$ & $39,3 \pm 1,33 \mathrm{AB} \mathrm{a}$ & $8,2 \pm 0,33 \mathrm{~B} \mathrm{~b}$ & $60,9 \pm 2,30 \mathrm{~B}$ \\
240 & $8,6 \pm 1,85 \mathrm{AB} \mathrm{b}$ & $39,8 \pm 3,38 \mathrm{AB} \mathrm{a}$ & $15,0 \pm 2,33 \mathrm{~B} \mathrm{~b}$ & $63,4 \pm 2,88 \mathrm{~B}$ \\
320 & $1,0 \pm 0,00 \mathrm{C} \mathrm{b}$ & $3,1 \pm 1,52 \mathrm{C} \mathrm{b}$ & $27,3 \pm 2,33 \mathrm{~A} \mathrm{a}$ & $31,4 \pm 2,02 \mathrm{C}$ \\
400 & $1,0 \pm 0,00 \mathrm{C} \mathrm{c}$ & $5,3 \pm 2,00 \mathrm{C} \mathrm{b}$ & $12,2 \pm 1,15 \mathrm{~B} \mathrm{a}$ & $18,5 \pm 1,20 \mathrm{C}$ \\
\hline C.V.1 $=22,7 \%$ & & & C.V. $2=24,8 \%$ \\
\hline
\end{tabular}

Dados originais apresentados para análise estatística os fatos foram transformados em arcsen $\sqrt{x / 100}$ Médias $( \pm \mathrm{EP})$ seguidas pela mesma letra minúscula na linha, e maiúscula na coluna não diferem entre si, pelo teste de Tukey $(\mathrm{P}<0,05)$.

Estudos realizados por Stathers, Mvumi e Golob (2002) avaliaram a persistência da TD contra pragas de grãos armazenados, e verificaram que os produtos testados apresentaram eficiência na proteção dos grãos por até quatro meses. Pinto Júnior (1999), em seus trabalhos, obteve proteção dos grãos contra o ataque de $S$. oryzae e $S$. zeamais (Motschulsky, $1855)$ por até sete meses, bem como Pinto Júnior, Lazzari e Lazzari (2005) verificaram até cinco meses de atividade contra Acanthoscelides obtectus
(Say, 1831) (Coleoptera: Bruchidae). No presente trabalho, valores satisfatórios de mortalidade foram alcançados até oito meses após a aplicação, porém, devem-se considerar as diferenças entre o ambiente deste estudo e o utilizado pelos referidos autores, que avaliaram insetos praga de grãos armazenados.

Verificou-se que até a quarta avaliação (240 dias), os maiores valores de mortalidade encontrados foram com 20 dias de exposição dos insetos à cama tratada. 
Porém, nas duas últimas avaliações, verificaram-se maiores valores de mortalidade somente após 30 dias de exposição (Tabela 1).

Segundo Pinto Júnior, Lazzari e Lazzari (2005), a eficácia dos tratamentos com TD está intimamente ligada às diferentes concentrações aplicadas, condições de incubação, localidades de obtenção do produto, mas principalmente ao tempo de contato/ dias de exposição e suscetibilidade das pragas. Esta suscetibilidade está relacionada à tolerância a dessecação (ou ao baixo volume de água interna), decorrente da composição lipídica do tegumento, capacidade de reabsorção de água do ambiente e relação área/volume da superfície do corpo do inseto, sendo que quanto menor o inseto, mais suscetível à ação dessecante da TD ele está (CHAPMAN, 1998; RIGAUX; HAUBRUGE; FIELDS, 2001).

Neste sentido, comparando insetos-alvo, Athanassiou, KavallieratoseAndris(2004)obtiveram um pico de mortalidade de $T$. confusum (Jacquelin du Val, 1868) após sete dias de exposição, sendo que neste mesmo período em um teste paralelo ocorreu mortalidade de $100 \%$ de $S$. oryzae. Por outro lado, Pinto Júnior, Lazzari e Lazzari (2005) avaliando a mortalidade de $A$. obtectus tratados com TD obteve índices de mortalidades de até $100 \%$ após apenas quatro dias, dependendo da concentração utilizada.

Considerando que as diferentes pragas receberam a TD nas respectivas concentrações recomendas para seu controle, a variação constatada entre o cascudinho e os insetos citados anteriormente, em relação ao período mínimo de contato para ocorrência dos maiores percentuais de mortalidade pode ser atribuída à superfície corpórea, uma vez que o cascudinho é maior em relação aos demais insetos citados, e também se encontram em um ambiente diferente.
Neste sentido, Faulde, Scharninghausen e Cavaljuga (2006) avaliaram a eficiência de TD para o controle de três espécies de baratas, obtendo picos de mortalidade diferenciados em relação ao cascudinho, variando entre seis, oito e nove dias de exposição ao tratamento. Neste caso, apesar das baratas serem maiores que o cascudinho, elas foram especialmente suscetíveis à ação da TD, pois possuem uma camada de lipídios na cutícula que aumenta o poder de adesão e adsorção das partículas do pó (EBELING, 1971).

Apesar de o presente estudo ter evidenciado a persistência da TD na cama de aviário, são necessários testes acerca dos possíveis efeitos que os fatores ambientais encontrados no campo podem ocasionar na eficácia da TD, visto que o aviário é um ambiente muito mais instável que as unidades de armazenamento, e possivelmente pode requerer o uso de uma concentração maior do produto ou de uma estratégia de aplicação diferenciada.

\section{Repelência}

Observou-se que a quantidade de insetos amostrados na área tratada com TD foi significativamente menor que a não tratada, apresentando, respectivamente $16,5 \%$ e $83,5 \%$ dos insetos coletados

Comparando-se o número de insetos nos diferentes períodos de avaliação, verificou-se que não houve efeito do tempo no número de insetos em cada área, porém, o percentual encontrado na área não-tratada sempre foi significativamente superior ao encontrado na área tratada, indicando ação repelente do produto (Tabela 2). 
Tabela 2. Porcentagem de adultos de $A$. diaperinus coletados na cama-de-aviário tratada com Terra de Diatomácea (TD) e não tratada (T), em diferentes dias após a liberação dos insetos $\left(26 \pm 2^{\circ} \mathrm{C}, 14 \mathrm{~h}\right.$ de fotofase $)$.

\begin{tabular}{ccccc}
\hline \multirow{2}{*}{ Tratamentos } & \multicolumn{3}{c}{ Dias após a liberação dos insetos } & \multirow{2}{*}{ Média } \\
\cline { 2 - 4 } & 1 & 4 & 7 & \\
\hline TD & $11,9 \pm 0,06 \mathrm{~B} \mathrm{a}$ & $15,3 \pm 0,06 \mathrm{~B} \mathrm{a}$ & $22,3 \pm 0,09 \mathrm{~B} \mathrm{a}$ & $16,5 \mathrm{~B}$ \\
T & $88,1 \pm 0,05 \mathrm{~A} \mathrm{a}$ & $84,7 \pm 0,05 \mathrm{~A} \mathrm{a}$ & $77,7 \pm 0,09 \mathrm{~A} \mathrm{a}$ & $83,5 \mathrm{~A}$ \\
\hline CV1 $(\%)=0,56$ & & $\mathrm{CV} 2(\%)=29,27$ &
\end{tabular}

Dados originais apresentados. Médias ( $\pm \mathrm{EP})$ seguidas pela mesma letra maiúscula na coluna e minúscula na linha não diferem entre si, segundo o teste de Tukey $(\mathrm{P}<0,05)$.

$\mathrm{CV} 1=$ Tratamento e CV2 $=$ Tempo

De acordo com El-Awami e Dent (1995), as baratas (Blattella germanica) que foram expostas às partículas de TD de tamanho $0,5-5 \mu \mathrm{m}$, foram as que mais apresentaram a atividade de limpeza, tanto das pernas como das antenas, em relação àquelas expostas às partículas maiores. Isso mostra que as menores partículas são mais incômodas para o inseto, apresentando maior chance de entrarem nos interstícios das articulações e penetrarem na cutícula e/ou nos espiráculos. Assim, como as partículas do produto usado neste experimento possuem até $5 \mu \mathrm{m}$ de tamanho, elas se enquadram neste perfil, possivelmente fazendo com que o inseto perceba a presença da TD no substrato por meio de órgãos sensoriais localizados em tais apêndices, quando entra em contato com o substrato tratado, evitando esta área.

Segundo White et al. (1966) a TD causa repelência devido à ação física, e a manutenção desta capacidade durante o período avaliado pode também ser atribuída às propriedades físicas das partículas, as quais são capazes de adsorver moléculas lipídicas da epicutícula dos insetos (EBELING, 1971) e continuam agindo enquanto o ambiente estiver seco e ainda houver concentração o suficiente de TD para que o inseto entre em contato com a mesma (KORUNIC, 1998).

Neste sentido, de acordo com Fields e Korunic (2000), a alta umidade do substrato interfere no modo de ação da TD, diminuindo a abrasão e conseqüentemente minimizando o efeito dessecante, sendo que segundo Vayas e Athanassiou (2004) 10\% de variação na umidade é suficiente para que ocorra uma redução considerável na mortalidade causada pela TD.

Porém, no presente estudo, verificou-se que não houve grande variação na umidade da cama, ao ponto que pudesse vir a comprometer a atividade da $\mathrm{TD}$, sendo que os valores encontrados na testemunha e no tratamento estiveram próximos em todas as avaliações, diferindo significativamente apenas na avaliação realizada 1 dia após a infestação (Tabela 3). 
Tabela 3. Porcentagem média de umidade da cama de aviário tratada e não tratada com Terra de Diatomácea (TD), em diferentes dias de avaliação após a aplicação.

\begin{tabular}{|c|c|c|}
\hline \multirow{2}{*}{ Avaliações (Dias) } & \multicolumn{2}{|c|}{ Tratamentos } \\
\hline & $\mathrm{TD}$ & Testemunha \\
\hline 1 & $30,7 \mathrm{~A} \mathrm{a}$ & $28,7 \mathrm{~A} \mathrm{~b}$ \\
\hline 4 & $23,8 \mathrm{~B} \mathrm{a}$ & $22,8 \mathrm{~B} \mathrm{a}$ \\
\hline 7 & $15,8 \mathrm{C} \mathrm{a}$ & $15,7 \mathrm{C} \mathrm{a}$ \\
\hline CV $1(\%)$ & 4,08 & \\
\hline CV $2(\%)$ & 2,68 & \\
\hline
\end{tabular}

Médias seguidas pela mesma letra minúscula na linha, e maiúscula na coluna não diferem entre si, pelo teste de Tukey $(\mathrm{P}<0,05)$.

$\mathrm{CV} 1=$ Tratamento $; \mathrm{CV}=$ Tempo

É provável que os valores de umidade da cama não tenham sido grandes o suficiente para interferir na ação da TD, tanto inseticida como repelente, como pode ser observado neste estudo.

Considerando-se a repelência da TD é verificada imediatamente à aplicação e contato com o inseto, e que de certa forma sofre pouca influência do tempo após o tratamento, se o substrato apresentar variação de umidade que reduza a ação inseticida, é possível ainda obter-se um efeito secundário (repelência), que contribui para o manejo do cascudinho no aviário.

Contudo, no desenvolvimento de estratégias de aplicação da TD no aviário, deve-se considerar este efeito, visto que na possibilidade de se realizar o tratamento em apenas algumas áreas do aviário, os insetos poderão se concentrarão em locais não tratados. Neste caso, outros métodos de controle deverão ser aplicados para evitar que os insetos permaneçam vivos e sejam consumidos pelas aves. Além disso, este efeito permite que a TD venha a ser utilizada como barreira para conter a migração dos insetos no aviário e impede que venha a ser utilizada na forma de isca tóxica contra a praga em questão.

\section{Referências}

ALVES, L. F. A.; BUZARELLO, G. D.; OLIVEIRA, D. G. P.; ALVES, S. B. Ação da terra de diatomácea contra adultos do cascudinho Alphitobius diaperinus (Panzer, 1797) (Coleoptera: Tenebrionidae). Arquivos do Instituto Biológico, São Paulo, v. 73, p. 115-118, 2006.

ALVES, V. S.; ALVES, L. F. A.; ROHDE, C. Patogenicidade de Steinernema glaseri e Steinernema carpocapsae (Nematoda: Rhabdita) contra o cascudinho, Alphitobius diaperinus (Panzer) (Coleoptera: Tenebrionidae). Neotropical Entomology, Londrina, v. 34, n. 1, p. 139-141, 2005.

ATHANASSIOU, C. G.; KAVALLIERATOS, N. G.; ANDRIS, N. S. Insecticidal effect of three diatomaceous earth formulations, applied alone or in combination, against three stored-product beetle species on wheat and maize. Journal of Stored Products Research, Oxford, v. 43, n. 4, p. 330-334, 2004.

BATES, C.; HIETT, K. L.; STERN, N. J. Relationship of Campylobacter isolated from poultry and from darkling beetles in New Zealand. Avian Diseases, Kennett Square, Pa., US, v. 48, p. 138-147, 2004.

CHAPMAN, R. F. The insects: structure and function. 4.ed. New York: Cambridge University Press, 1998.

CHERNAKI-LEFFER, A. M. Dinâmica populacional, estimativa da resistência a inseticidas e alternativas de controle para o cascudinho Alphitobius diaperinus (Panzer, 1797) (Coleoptera: Tenebrionidae). 2004. Tese (Doutorado) - Programa de Pós-Graduação em Entomologia, Universidade Federal do Paraná, Curitiba. 
CHERNAKI-LEFFER, A. M.; BIESDORF, S. M.; ALMEIDA, L. M.; LEFFER, E. V. B.; VIGNE, F. Isolamento de enterobactérias em Alphitobius diaperinus e na cama de aviários no oeste do estado do Paraná, Brasil. Revista Brasileira de Ciência Avícola, Campinas, v. 4, n. 3, p. 243-247, 2002.

CHERNAKI-LEFFER, A. M.; LAZZARI, F. A.; LAZZARI, S. M. N.; ALMEIDA, L. M. Controle do cascudinho. Avicultura Industrial, São Paulo, n. 1094, p. 22-25, 2001.

DESPINS, J. L.; AXTELL, R. C. Feeding behavior and growth of broiler chicks fed larvae of the darkling beetle, Alphitobius diaperinus. Poultry Science, Champaign, v. 74, p. 331-336, 1995.

EBELING, W. Sorptive dusts for pests control. Annual Review of Entomology, Stanford, v. 16, p. 123-158, 1971.

EL-AWAMI, I. O.; DENT, D. R.. The interaction of surface and dust particle size on the pick-up and grooming behavior of the German cockroach Blattella germanica. Entomologia Experimentalis et Applicata, Dordrecht, Holanda, v. 77, p. 81-81, 1995.

EMPRESA BRASILEIRA DE PESQUISA AGROPECUÁRIA - EMBRAPA. Centro Nacional de Pesquisa de Solos (Rio de Janeiro, RJ). Manual de métodos de análise de solo. Rio de Janeiro: EMBRAPACNPS, 1997. (Documentos 1).

FAULDE, M. K.; SCHARNINGHAUSEN, J. J.; CAVALJUGA, S. Efficacy of modified diatomaceous earth on different cockroach species (Orthoptera, Blattellidae) and silverfish (Thysanura, Lepismatidae). Journal of Pesticide Science, Tokyo, v. 79, n. 3, p. 155161, 2006.

FERREIRA, D. F. SISVAR (Sistema para análise de variância para dados balanceados). Lavras: UFLA, 1992. Disponível em: <http://www.dex.ufla.br/danielff/ dff02.htm>. Acesso em: 23 dez. 2005.

FIELDS, P.G.; KORUNIC, Z. The effect of grain moisture content and temperature on the efficacy of diatomaceous earths from different geographical locations against stored-product beetles. Journal of Stored Products Research, Oxford, v. 36, p. 1-13, 2000.

KORUNIC, Z. Diatomaceous earths, a group of natural insecticides. Journal of Stored Products Research, Oxford, v. 34, n. 2-3, p. 87-97, 1998.

LORINI, I.; FERREIRA-FILHO, A.; BARBIERI, I.; DEMAMAN, N. A.; MARTINS, R. R.; DALBELLO, O. Terra de diatomáceas como alternativa no controle de pragas de milho armazenado em propriedade familiar.
Agroecologia e Desenvolvimento Rural Sustententável, Porto Alegre, v. 2, n. 4, p. 32-36, 2001.

MARCOMINI, A. Atividade inseticida de diferentes extratos vegetais sobre adultos do cascudinho, Alphitobius diaperinus Panzer (Coleoptera, Tenebrionidae), em condições de laboratório. 2006. Trabalho de Conclusão de Curso (Graduação em Ciências Biológicas) Universidade Estadual do Oeste do Paraná, Cascavel,PR, 2006.

MCALLISTER, J. C.; STEELMAN, C. D.; NEWBERRY, L. A.; SKEELES, J. K. Isolation of infectious bursal disease virus from the lesser mealworm, Alphitobius diaperinus (Panzer). Poultry Science, Champaign, v. 74, p. 45-49, 1995.

MOHAN, S.; FIELDS, P. G. A simple technique to assess compounds that are repellent or attractive to storedproduct insects. Journal of Stored Products Research, Oxford, n. 38, p. 23-31, 2002.

OLIVEIRA, D. G. P.; ALVES, L. F. A. Interação do fungo Beauveria bassiana (Bals.) Vuill. com terra diatomácea para o controle de Alphitobius diaperinus (Panzer) (Coleoptera: Tenebrionidae), o cascudinho dos aviários. BioAssay, Piracicaba, SP, v. 2, p. 1-6, 2007.

PINTO JÚNIOR, A. R.; LAZZARI, F. A.; LAZZARI, S. M. N. Controle de Acanthoscelides Obtectus (Coleóptera: Bruchidae) com diferentes doses de Terra Diatomácea (Dióxido de Sílica). Revista Acadêmica: Ciências Agrárias e Ambientais, Curitiba, v. 3, n. 1, p. 75-79, 2005.

PINTO JÚNIOR. A. R. Utilização de terra de diatomácea no controle de pragas de grãos armazenados $e$ domissanitárias. 1999. Tese (Doutorado) - Programa de Pós-Graduação em Entomologia, Universidade Federal do Paraná, Curitiba.

QUARLES, W. Diatomaceous earth for pest control. IPM Practitioner, New York, v. 14, n. 5-6, p. 1-11, 1992.

RIGAUX, M.; HAUBRUGE, E.; FIELDS, P.G. Mechanisms for tolerance to diatomaceous earth between strains of Tribolium castaneum. Entomologia Experimentalis et Applicata, Dordrecht, Holanda, v. 101, p. 33-39, 2001.

ROHDE, C.; ALVES, L. F. A.; NEVES, P. M. O. J.; ALVES, S. B.; SILVA, E. R. L.; ALMEIDA, J. E. M. Seleção de isolados de Beauveria bassiana (Bals.) Vuill. e Metarhizium anisopliae (Metsch.) Sorok contra o cascudinho Alphitobius diaperinus (Panzer) (Coleoptera: Tenebrionidae). Neotropical Entomology, Londrina, v. 35, p. 231-240, 2006. 
SANTORO, P. H.; NEVES, P. M. O. J.; CAVAGUCHI, S. A.; CONSTANSKI, K.; AMARO, J. T.; ALVES, L. F. A.; GOMES, B. B. Controle associado de Alphitobius diaperinus e efeito de microrganismos eficazes no desenvolvimento de Beauveria bassiana. Pesquisa Agropecuária Brasileira, Rio de Janeiro, v. 43, n. 1, p. 1-8, 2008.

STATHERS, T. E.; MVUMI, B. M.; GOLOB, P. Field assessment of the efficacy and persistence of diatomaceous earths in protecting stored grain on small-scale farms in Zimbabwe. Crop Protection, Guildford, Inglaterra, v. 21, p. 1033-1048, 2002.
STEELMAN, D. Darkling beetles are costly pests. Poultry Digest, Mount Morris, v. 55, p. 22-23, 1996.

VAYAS, B. J.; ATHANASSIOU, C. G. Factors affecting the insecticidal efficacy of the diatomaceous earth formulation SilicoSec against adults and larvae of the confused flour beetle, Tribolium confusum DuVal (Coleoptera: Tenebrionidae). Crop Protection, Guildford, Inglaterra, v. 23, p. 565-573, 2004.

WHITE, G. D.; BERNDT, W. L.; SCHESSER, J. H.; FIFIELD, C. C. Evaluation of four inert dusts for the protection of stored wheat in Kansas from insect attack. USDA: Agricultural Research Service Report, Washington, v. 58, n. 8, p.1-21, 1966. 
